\title{
Trombosis cava con extensión auricular en un paciente con hepatocarcinoma
}

\author{
Inferior vena cava thrombosis with atrial extension in a patient \\ with hepatocellular carcinoma
}

Diego Aguirre', Laura Bernal2 ${ }^{2}$,Hernán Carranza ${ }^{3,4}$, Carlos Vargas ${ }^{3,4}$, Jorge Miguel Otero ${ }^{3,4}$, Belén de Molano ${ }^{5}$,
Andrés Felipe Cardona ${ }^{2-4}$

'Departamento de Imágenes Diagnósticas, Sección Imagen Corporal, Fundación Santa Fe de Bogotá (Bogotá, Colombia)

¿Sección Investigación Clínica y Traslacional, Instituto de Oncología, Fundación Santa Fe de Bogotá (Bogotá, Colombia).

Grupo Oncología Clínica y Traslacional, Fundación Santa Fe de Bogotá (Bogotá, Colombia).

"Fundación para la Investigación Clínica y Molecular Aplicada del Cáncer (FICMAC) (Bogotá, Colombia).

El hepatocarcinoma (HC) es una neoplasia con cierta propensión a invadir estructuras vasculares, como la vena porta y la hepática, donde causa trombosis tumoral, hallazgo que representa un importante predictor de mortalidad. La frecuencia de invasión vascular se ha estimado en el 7,5\%, aunque algunas series han informado frecuencias que oscilan alrededor del $30 \%$ en autopsias'. La frecuencia aumenta de forma significativa según el tamaño tumoral², y, en algunos casos, el trombo tumoral de la vena hepática puede extenderse hasta la cava inferior ( $\mathrm{VCI}$ ) y a la aurícula derecha, evento reportado entre el 3,8 y $6 \%$, y el 2 y $11 \%$, respectivamente ${ }^{3-4}$. La presencia de trombosis es mayor en pacientes con $\mathrm{HC}$ localmente avanzado o metastásico. No obstante, la trombosis tumoral con extensión a la $\mathrm{VCl}$ y a la aurícula derecha es bastante rara, ya que se han reportado menos de 300 casos.

En general, el pronóstico de pacientes que presentan esta condición es bastante pobre, con una mediana de supervivencia global (SG) de 1,9 meses y una supervivencia estimada a 6 y 12 meses del 24 y $0 \%$, respectivamente. Si la obstrucción de la $\mathrm{VCl}$ es total, se puede desarrollar un síndrome de Budd-Chiari secundario, pero, en el caso contrario, puede no haber síntomas ni signos clínicos específicos, por lo que la mayoría de los diagnósticos se realizan post mórtem. Con los avances tecnológicos en imaginología, el reconocimiento de esta condición clínica ha venido en aumento, permitiendo reconocer otras complicaciones, como el infarto pulmonar y la falla cardíaca, que se asocia a muerte súbita en presencia de obstrucción aguda de la válvula tricúspide, con compromiso del tracto de salida ventricular o embolismo pulmonar ${ }^{6}$.
La literatura es escasa respecto de la fisiopatología de la trombosis tumoral en el HC. Entre las alteraciones encontradas, se han identificado algunas moléculas de adhesión, como MT1-MMP y MMP-2, y se ha observado sobreexpresión del factor tisular (FT). Este último ha sido identificado en células tumorales de HC y representa un mecanismo importante implicado en el desarrollo de un estado hipercoagulable que promueve la trombosis local y sistémica ${ }^{7}$. El FT induce la migración y adhesión celular, y promueve la angiogénesis, en parte mediada por el factor de crecimiento derivado del endotelio vascular (vascular endothelial groeth factor, VEGF), que, a su vez, contribuye con la progresión y la metástasis. Las proteínas que se generan por la activación del sistema de coagulación en respuesta al daño tisular coadyuvan con la angiogénesis propia de la reparación tisular.

Esta neovascularización activa es un mecanismo identificado de progresión en el HC, donde provee oxígeno y nutrientes, facilitando el crecimiento tumoral y una ruta para la invasión del sistema circulatorio. Así, los niveles de expresión del FT se han correlacionado positivamente con la presencia de invasión venosa y trombosis tumoral'8; esto podría sugerir que los valores de FT sirven para determinar la progresión tumoral de forma independiente, sin embargo, se requieren más estudios para aclarar su valor predictivo y pronóstico ${ }^{9,10}$. Por otro lado, los niveles de VEGF parecen útiles para predecir la invasión venosa por el HC, encontrando también deficiencia de proteína C, S y antitrombina III, incluso en ausencia de enfermedad hepática preexistente.

Varios estudios han establecido que el tamaño tumoral y el nivel de alfa fetoproteína (AFP) son 


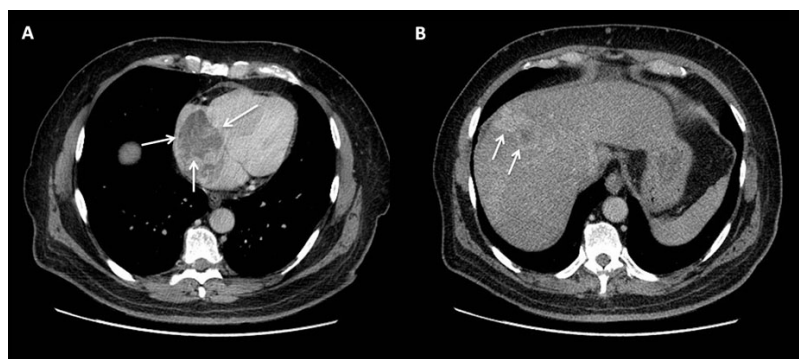

Figura 1. Imágenes de escanografía con contraste, en bases pulmonares (A), demuestran trombo endoluminal en aurícula derecha (flechas), heterogéneo. En la imagen a la altura del hígado (B), se observa lesión focal irregular, hipervascular, en lóbulo derecho (flechas), sospechosa de lesión neoplásica.

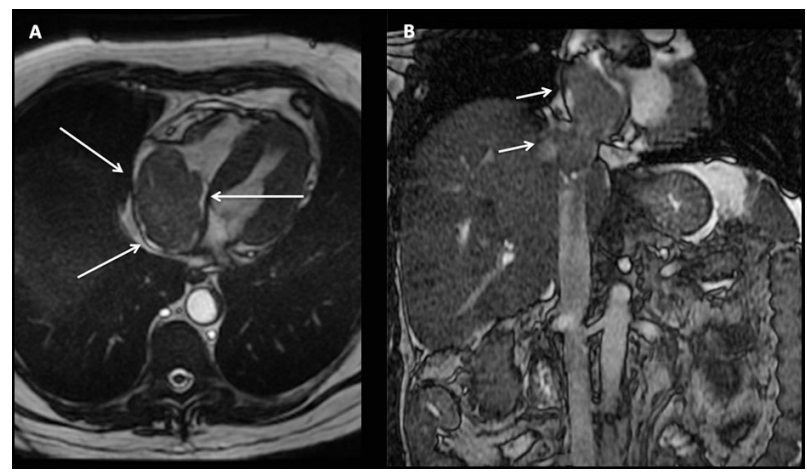

Figura 3. Imágenes por resonancia magnética cardíaca en los planos axial (A) y coronal (B), secuencia Fiesta, que demuestran trombo endoluminal en aurícula derecha, el cual se extiende desde la vena cava inferior porción hepática (flechas).

Figura 4. Imágenes de PET/CT 18-FDG en los planos coronal y axial (A y B), que demuestran lesión hipermetabólica que se extiende desde el parénquima hepático, compromete la vana cava inferior, hasta la aurícula derecha, correspondiente a carcinoma hepatocelular, con trombosis tumoral de venas hepática, vena cava inferior y aurícula derecha.

predictores de la presencia o ausencia de invasión vascular. Se ha mostrado mayor incidencia de trombosis tumoral en pacientes con niveles de AFP superiores a $1.000 \mathrm{ng} / \mathrm{ml}^{2}$. Koo y colaboradores describieron un posible mecanismo fisiopatológico de tipo mecánico, donde la obstrucción del flujo sanguíneo generada por la compresión tumoral contribuye con el desarrollo de un estado protrombótico ${ }^{4}$. Por último, se ha establecido una clara relación entre la infección por el virus de hepatitis $B(\mathrm{VHB})$, la coinfección por hepatitis $C$ y el desarrollo de trombosis tumoral.

El proceso carcinogénico iniciado por el VHB se da a través de la instauración de un estado inflamatorio procoagulante, con extensa activación de múltiples citoquinas. Estos cambios patológicos contribuyen con la trombosis tumoral, que se asocia con el aumento en los niveles de ADN circulante del VHB. Un estudio publicado recientemente demostró en pacientes VHB

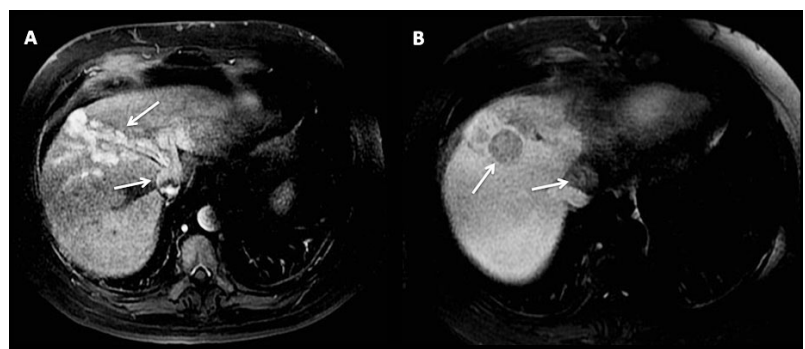

Figura 2. Imágenes del abdomen superior por resonancia magnética, con información gradiente de eco, posterior a la administración de medio de contraste paramagnético, durante la fase arterial (A) y venosa portal (B), que demuestran lesión hipervascular, con compromiso de venas hepáticas media e izquierda por trombo tumoral, con extensión a vena cava inferior (flechas). Obsérvese que la lesión nodular dominante presenta lavado de contraste en la fase portal, al igual que el trombo tumoral de vena cava inferior (flechas).

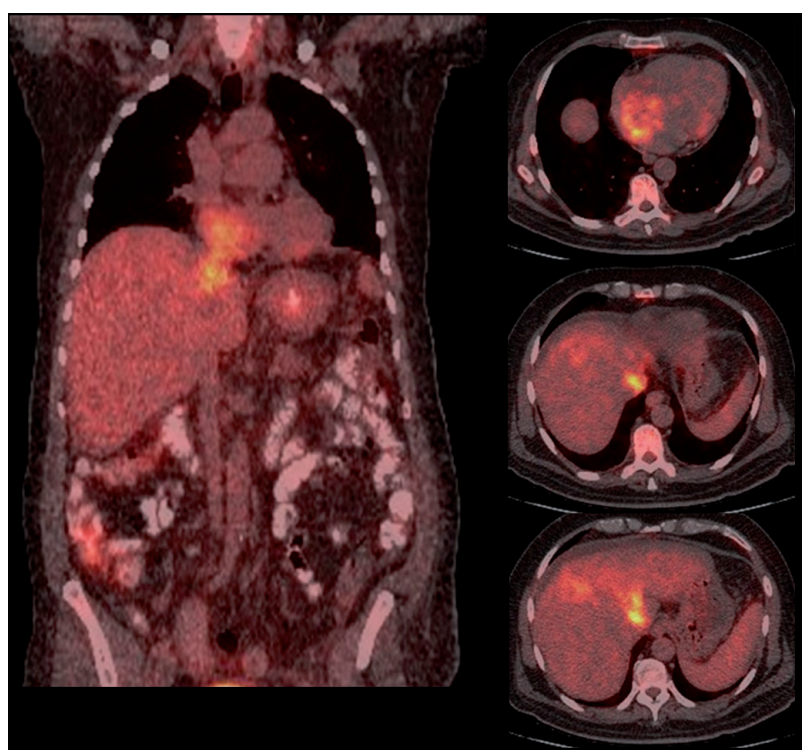

positivos la activación de la vía TGF $\beta$-miR-34a-CCL22, responsable de la inmunosupresión en el microambiente tumoral y en el sistema venoso circundante, que favorece la diseminación de las células anormales ${ }^{11}$. También, se han hallado niveles alterados de micro-RNA resultantes de la expresión aberrante de algunos genes, que contribuyen con el desarrollo de metástasis, incluyendo el micro-RNA-135a y el FOXM1-miR-135a-MTSS12.

La remoción quirúrgica del trombo tumoral es técnicamente difícil y altamente mórbida; de igual forma, la mortalidad informada después de este procedimiento excede el $15 \%$ en las cuatro semanas siguientes a la intervención. La dificultad es mayor, considerando que los pacientes se encuentran en un estado avanzado de la enfermedad, con cirrosis grave y una reserva funcional hepática limitada. Sin embargo, la hepatectomía radical más trombectomía 
se realiza con cierta frecuencia luego del desarrollo de la técnica de exclusión vascular hepática total (THVE, por su sigla en inglés) ${ }^{13}$. Aunque este procedimiento sea exitoso, el trombo recurre en el $30 \%$ de los casos, por lo que siempre se requiere terapia anticoagulante, que no modifica la supervivencia reportada entre los 8 y 12 meses.

\section{Referencias}

1. Inoue $Y$, Hayashi $M$, Katsumata $T$, Shibayama $Y$, Tanigawa $N$. Hepatocellular carcinoma with right atrial tumor thrombus: report of a case. Surgery Today. 2011;41(8):1122-9.

2. Panduranga P, Al-Mukhaini M, Ratnam L, Al-Harthy S. Mobile right atrial thrombus with pulmonary thromboembolism in a patient with advanced hepatocellular carcinoma and disseminated tumor thrombosis. Heart Views. 2011;12(4):173-7.

3. Georgen M, Regimbeau J-M, Kianmanesh R, Marty J, Farges $\mathrm{O}$, Belghiti J. Removal of hepatocellular carcinoma extending in the right atrium without extracorporal bypass. J Am Coll Surg. 2002;195(6):892-4

4. Sung AD, Cheng S, Moslehi J, Scully EP, Prior JM, Loscalzo J. Hepatocellular carcinoma with intracavitary cardiac involvement: a case report and review of the literature. Am J Cardiol. 2008;102(5):643-5

5. Tse HF, Lau CP, Lau YK, Lai CL. Transesophageal echocardiography in the detection of inferior vena cava and cardiac metastasis in hepatocellular carcinoma. Clin Cardiol. 1996;19(3):211-3.

6. Lin HH, Hsieh CB, Chu HC, Chang WK, Chao YC, Hsieh TY. Acute pulmonary embolism as the first manifestation of hepatoceIlular carcinoma complicated with tumor thrombi in the inferior vena cava: surgery or not? Dig Dis Sci. 2007;52(6):1554-7.
7. Masci G, Magagnoli M, Grimaldi A, Covini G, Carnaghi C, Rimassa L, et al. Metastasis of hepatocellular carcinoma to the heart: a case report and review of the literature. Tumori. 2004;90(3):345-7.

8. Poon RT, Lau CP, Ho JW, Yu W, Fan S, Wong J. Tissue factor expression correlates with tumor angiogenesis and invasiveness in human hepatocellular carcinoma. Clin Cancer Res. 2003;9(14):5339-45.

9. Connolly GC, Chen R, Hyrien $O$, Mantry P, Bozorgzadeh $A$, Abt $P$, et al. Incidence, risk factors and consequences of portal vein and systemic thromboses in hepatocellular carcinoma. Thromb Res. 2008;122(3):299-306.

10. Belting $M$, Ahamed J, Ruf W. Signaling of the tissue factor coagulation pathway in angiogenesis and cancer. Arterioscler Thromb Vasc Biol. 2005;25(8):1545-50.

11. Yang P, Li Q, Feng Y, Zhang Y, Markowitz GJ, Ning S, et al. TGFb-miR-34a-CCL22 signaling-induced Treg cell recruitment promotes venous metastases of HBV-positive hepatocellular carcinoma. Cancer Cell. 2012;22(3):291-303.

12. Liu S, Guo W, Shi J, Li N, Yu X, Xue J, et al. MicroRNA-135a contributes to the development of portal vein tumor thrombus by promoting metastasis in hepatocellular carcinoma. J Hepatol. 2012;56(2):389-96.

13. Li AJ, Zhou WP, Lin C, Lang $X L$, Wang ZG, Yang $X Y$, et al. Surgical treatment of hepatocellular carcinoma with inferior vena cava tumor thrombus: a new classification for surgical guidance. Hepatobiliary Pancreat Dis Int. 2013;12(3):263-9.

14. Liu J, Wang Y, Zhang D, Liu B, Ou Q. Comparison of survival and quality of life of hepatectomy and thrombectomy using total hepatic vascular exclusion and chemotherapy alone in patients with hepatocellular carcinoma and tumor thrombi in the inferior vena cava and hepatic vein. Eur J Gastroenterol Hepatol. 2012;24(2):186-94. 